\title{
Evolution of the ion environment of comet 67P/Churyumov-Gerasimenko
}

\section{Observations between 3.6 and 2.0 AU}

H. Nilsson ${ }^{1,2}$, G. Stenberg Wieser ${ }^{1}$, E. Behar ${ }^{1,2}$, C. Simon Wedlund ${ }^{3}$, E. Kallio ${ }^{3}$, H. Gunell ${ }^{4}$, N. J. T. Edberg ${ }^{5}$, A. I. Eriksson ${ }^{5}$, M. Yamauchi ${ }^{1}$, C. Koenders ${ }^{6}$, M. Wieser ${ }^{1}$, R. Lundin ${ }^{1}$, S. Barabash ${ }^{1}$, K. Mandt ${ }^{7}$, J. L. Burch ${ }^{7}$, R. Goldstein ${ }^{7}$, P. Mokashi ${ }^{7}$, C. Carr ${ }^{8}$, E. Cupido ${ }^{8}$, P. T. Fox ${ }^{8}$, K. Szego ${ }^{9}$, Z. Nemeth ${ }^{9}$, A. Fedorov ${ }^{10}$, J.-A. Sauvaud ${ }^{10}$, H. Koskinen ${ }^{11,12}$, I. Richter ${ }^{6}$, J.-P. Lebreton ${ }^{13}$, P. Henri ${ }^{13}$, M. Volwerk ${ }^{14}$, C. Vallat ${ }^{15}$, and B. Geiger ${ }^{15}$

1 Swedish Institute of Space Physics, Box 812, 98128 Kiruna, Sweden e-mail: hans.nilsson@irf.se

2 Luleå University of Technology, Department of Computer Science, Electrical and Space Engineering, Rymdcampus 1, 98128 Kiruna, Sweden

3 Aalto University, School of Electrical Engineering, Department of Radio Science and Engineering, PO Box 13000, 00076 Aalto, Finland

4 Belgian Institute for Space Aeronomy, avenue Circulaire 3, 1180 Brussels, Belgium

5 Swedish Institute of Space Physics, Ångström Laboratory, Lägerhyddsvägen 1, Uppsala, Sweden

6 TU - Braunschweig, Institute for Geophysics and extraterrestrial Physics, Mendelssohnstr. 3, 38106 Braunschweig, Germany

7 Southwest Research Institute, 6220 Culebra Rd., San Antonio, TX 78238, USA

8 Imperial College London, Exhibition Road, London SW7 2AZ, UK

9 Wigner Research Centre for Physics, 1121 Konkoly Thege street 29-33, Budapest, Hungary

${ }_{10}$ Institut de Recherche en Astrophysique et Planétologie, 31028 Toulouse, France

11 University of Helsinki, Department of Physics, PO Box 64, University of Helsinki, 00014 Helsinki, Finland

12 Finnish Meteorological Institute, PO BOX 503, 00101 Helsinki, Finland

13 Laboratoire de Physique et Chimie de l'Environnement et de l'Espace (LPC2E), UMR 7328 CNRS - Université d'Orléans, France

14 Space Research Institute, Austrian Academy of Sciences, Schmiedlstraße 6, 8042 Graz, Austria

15 Rosetta Science Ground Segment, SRE-OOR, Office A006, European Space Astronomy Centre, PO Box 78, 28691 Villanueva de la Cañada, Madrid, Spain

Received 20 March 2015 / Accepted 6 May 2015

\section{ABSTRACT}

\begin{abstract}
Context. The Rosetta spacecraft is escorting comet 67P/Churyumov-Gerasimenko from a heliocentric distance of $>3.6$ AU, where the comet activity was low, until perihelion at 1.24 AU. Initially, the solar wind permeates the thin comet atmosphere formed from sublimation.

Aims. Using the Rosetta Plasma Consortium Ion Composition Analyzer (RPC-ICA), we study the gradual evolution of the comet ion environment, from the first detectable traces of water ions to the stage where cometary water ions accelerated to about $1 \mathrm{keV}$ energy are abundant. We compare ion fluxes of solar wind and cometary origin.

Methods. RPC-ICA is an ion mass spectrometer measuring ions of solar wind and cometary origins in the $10 \mathrm{eV}-40 \mathrm{keV}$ energy range.

Results. We show how the flux of accelerated water ions with energies above $120 \mathrm{eV}$ increases between 3.6 and $2.0 \mathrm{AU}$. The $24 \mathrm{~h}$ average increases by 4 orders of magnitude, mainly because high-flux periods become more common. The water ion energy spectra also become broader with time. This may indicate a larger and more uniform source region. At 2.0 AU the accelerated water ion flux is frequently of the same order as the solar wind proton flux. Water ions of $120 \mathrm{eV}-\mathrm{few} \mathrm{keV}$ energy may thus constitute a significant part of the ions sputtering the nucleus surface. The ion density and mass in the comet vicinity is dominated by ions of cometary origin. The solar wind is deflected and the energy spectra broadened compared to an undisturbed solar wind.

Conclusions. The flux of accelerated water ions moving from the upstream direction back toward the nucleus is a strongly nonlinear function of the heliocentric distance.
\end{abstract}

Key words. plasmas - acceleration of particles - comets: general - comets: individual: 67P/Churyumov-Gerasimenko

\section{Introduction}

The ESA/Rosetta mission (Glassmeier et al. 2007) provides a unique opportunity to increase our knowledge about comets in general and about the Jupiter-family comet 67P/ChuryumovGerasimenko in particular. Comets are believed not to have changed much since the formation of the solar system and, hence, they are the most pristine celestial objects available to us. Comets are formed at large heliocentric distances, where the solar radiation is weak and the solar wind tenuous, and they interact sparsely with their environment. The surface is expected to be weathered for instance by cosmic rays. During their visits 
in the inner solar system, the Sun is able to affect the comets more strongly: Rosetta hence provides a unique opportunity to study the Sun-comet interaction mechanisms in detail.

When a comet approaches the Sun, material on its surface sublimates. A huge atmosphere of gas and dust forms, and the most distinctive feature of a comet appears: the comet tail. Long before it could be verified in situ, shapes and directions of comet ion tails suggested that comets interact with a continuous stream of plasma originating from the Sun (Biermann 1951; Alfvén 1957).

The interaction between a fully active comet and the solar wind resembles the interaction between the solar wind and an unmagnetized planet, such as Mars or Venus. A partly ionized, and thereby electrically conducting atmosphere, presents an obstacle to the solar wind flow. As a consequence, an induced magnetosphere is created when a (weak) bow shock and a contact surface form and the interplanetary magnetic field piles up on the sunward side of the comet (Cravens \& Gombosi 2004). The neutral particles in the comet's atmosphere are exposed to solar radiation and photoionize. Solar wind charge exchange and electron impact ionization can also play a major role in ion production. The newborn ions respond to the electric and magnetic fields in the solar wind and are "picked up" by the solar wind flow, that is, they are accelerated by the solar wind convective electric field. The energy required to incorporate the pickup ions in the flow is taken from the solar wind, which, as a result, may become deflected and slowed down, a mechanism known as mass loading (e.g., Coates 1997; Szegö et al. 2000). The cycloid paths traveled by the pickup ions in real space lead to the formation of ring distributions in velocity space if the the source region is larger than the ion gyroradius (Richardson et al. 1987; Coates et al. 1989). Such distributions are highly unstable and result in a rapid growth of low-frequency waves (Neugebauer 1990, and references therein).

At large distances from the Sun, however, the cometary atmosphere is still tenuous and the solar wind passes through undisturbed. The plasma pressure from the ions of cometary origin cannot compete with the dynamic pressure from the solar wind, and the solar wind ions impact the comet surface, causing sputtering (Coates 1997). Hybrid models indicate that we do not expect to see a bow shock or another plasma boundary in the early phase of the Rosetta mission (Rubin et al. 2014).

When the cometary atmosphere becomes sufficiently dense, collisions start to become important. Solar wind ions collide with molecules in the cometary atmosphere, and following chargeexchange processes, solar wind protons are converted into hydrogen atoms and solar wind $\mathrm{He}^{2+}$ ions become $\mathrm{He}^{+}$ions. Singly charged helium ions created in these charge-exchange processes are indeed observed by the ion spectrometers RPC-ICA (Nilsson et al. 2007) and RPC-IES (Burch et al. 2007) in the vicinity of $67 \mathrm{P} /$ Churyumov-Gerasimenko. The hydrogen atoms are not measured directly, but it is estimated that close to the comet nucleus more than $30 \%$ of the arriving solar wind protons have charge-exchanged into energetic neutral atoms (Nilsson et al. 2015).

As Rosetta escorts comet 67P/Churyumov-Gerasimenko on its journey through the inner parts of the solar system, the heliocentric distance changes from 3.6 AU, when Rosetta first arrived at a distance of $100 \mathrm{~km}$ from the nucleus, to $1.24 \mathrm{AU}$ at the comet's perihelion passage. The interaction between the cometary atmosphere and the solar wind is expected to evolve and intensify during this time. In this paper we use the mass-resolving ion spectrometer (RPC-ICA, Nilsson et al. 2007) onboard Rosetta to explore how the activity of
67P/Churyumov-Gerasimenko has changed as a function of the heliocentric distance. The very first observations of cometary water ions by the RPC-ICA were made on 7 August 2014 at a distance of $100 \mathrm{~km}$ from the nucleus (Nilsson et al. 2015). Shortly after these first cometary water ions were observed, strongly increased water ion fluxes and accelerated water ions with an energy of up to $800 \mathrm{eV}$ were observed. Detailed observations of the pick-up process have also been reported based on data from RPC-IES (Goldstein et al. 2015). Although the plasma boundaries have not yet formed, the comet's atmosphere is still able to significantly deflect the solar wind flow (Nilsson et al. 2015; Broiles et al. 2015). The solar wind magnetic field is also significantly affected by the comet interaction; strong wave activity at about $40 \mathrm{mHz}$, well above the local water ion gyrofrequency, has been observed since arrival at the comet (Richter et al. 2015).

We show the evolution of the observed comet water ions and solar wind ions between heliocentric distances of 3.6 down to 2.0 AU. We show all the data in one overview energy spectrogram, along with trends and statistics of the energetic ion environment of comet 67P/Churyumov-Gerasimenko.

\section{Instrument description}

The Rosetta Plasma Consortium Ion Composition Analyser (RPC-ICA) is an ion spectrometer with modest mass resolution capabilities (Nilsson et al. 2007). The instrument covers an energy range from $10 \mathrm{eV}$ up to $40 \mathrm{keV}$ per elementary charge (e) for positively charged ions. Ion energy is determined through an electrostatic analyzer with an energy resolution of $7 \%$. Mass resolution is achieved after the electrostatic analyzer through a system with permanent magnets that causes a spatial separation of ions of different mass. RPC-ICA can distinguish between mass groups of 1, 2, 4, 8, 16, and $32 \mathrm{amu} / \mathrm{e}$. A post-acceleration voltage is used after the electrostatic analyzer, and this affects the mass range and mass resolution of the instrument.

A full energy sweep is performed in $12 \mathrm{~s}$. The field of view is near $2.8 \pi \mathrm{sr}$, but is in practice closer to $2 \pi$ because of spacecraft shadowing. The basic instrument viewing plane is illustrated in Fig. 1. Angular resolution in the viewing plane is achieved through 16 anode sectors. The angular field of view of each sector is $22.5^{\circ} \times 4^{\circ}$. Deflection plates at the instrument entrance make it possible to increase the basic field of view. By changing the voltage on the plates, the instrument scans through 16 elevation angles in the range $\pm 40^{\circ}$ from the central viewing plane. A complete scan is achieved in $192 \mathrm{~s}$. At energies above $15 \mathrm{keV}$ the angular coverage is gradually decreased because the voltage is not high enough to cover the larger elevation defection angles. For energies below $200 \mathrm{eV}$, the resolution of the voltage setting is insufficient to set the desired angle exactly, leading to a decreased angular coverage.

The mass is distinguished through a 32 anode radial position system. Of these anodes, or mass channels, a total of eight are not giving a proper response. In particular, two mass channels just inside the main proton mass position, and four mass channels just outside the main proton mass position for average solar wind energy are not operational. Fortunately, two mass channels located where the peak of the proton population hits the detector surface for average solar wind energies and high postacceleration setting are working well.

Laboratory calibration shows that the shape of the proton distribution on the detector plate is expected to be similar to that for $\mathrm{He}^{2+}$ (Nilsson et al. 2007). The half-width of the $\mathrm{He}^{2+}$ response is observed to be four to six mass channels wide. We thus expect 


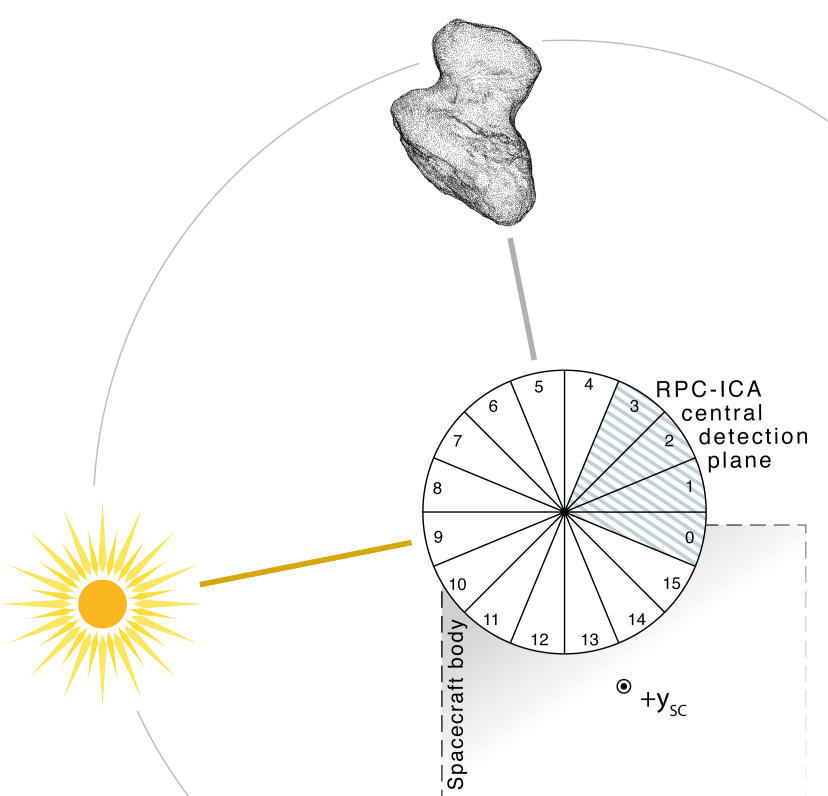

Fig. 1. ICA viewing geometry. Each sector is $22.5^{\circ}$ wide. The Sun is always within a few degrees of the depicted central viewing plane of RPC-ICA. The illustrated positions of the Sun and comet depict a common situation. Sectors 0 to 3 are not used in this study. They are indicated by a striped shading. The elevation angle of ICA denotes the angle of observed ions beyond the depicted central detector plane.

the proton distribution to be four to six mass channels wide as well. Sampling only the central two mass channels of the proton distribution will then lead to an underestimate of typically a factor 2. The same is not true for the $\mathrm{He}^{2+}$ particle flux, where all the mass channels corresponding to the main signal for typical solar wind energies are working well (see Nilsson et al. 2015, for an example of the mass response of the instrument).

After arrival at the comet, it was discovered that there is an offset in the voltage of the electrostatic analyzer. This offset also led to a mismatch between the elevation angle deflection voltage and the electrostatic analyzer voltage. This was remedied with a software patch effective from 30 October 2014. Data for energies below a few $100 \mathrm{eV}$ are strongly affected by this, and data from before and after the patch cannot be directly compared for energies below about $100 \mathrm{eV}$. For energies between $120 \mathrm{eV}$ and $550 \mathrm{eV}$ the angular range covered was similar before and after the patch, but the coverage was sparser before the patch. The energy coverage was also coarser. We have compensated for this in the statistics by multiplying the old data with a factor reflecting the number of elevation angle bins available in this energy range. To make a best possible comparison between the data before and after the patch, we have used $120 \mathrm{eV}$ as a lower limit for accelerated water ions. We have used $60 \mathrm{eV}$ as the upper limit for the cold ion population. Using $60 \mathrm{eV}$ as the lower limit for accelerated water ions does not change the conclusions of this paper. When calculating integrated fluxes, the coarser energy sampling of the pre-patch data may lead to both under- and overestimates of the actual flux.

Throughout the mission, the RPC-ICA instrument has suffered several sudden overheating events, where the instrument was automatically switched off. It was believed that these events presented a hazard to the instrument and therefore the instrument was used sparingly in the beginning of the mission. Since the beginning of September 2014, only two of these overheating events have occurred, leading to a gradual increase of the run time of the instrument. Since the beginning of 2015, the instrument is run full time except for periods around thruster firings.

Because of limited telemetry, RPC-ICA is frequently operated in a low-telemetry mode (normal mode) where the data are binned. The onboard mass lookup table used in normal mode is not optimal, but allows for a good separation between water ions and solar wind ions. In principle, the different solar wind ions are also quite well distinguished, but handling crosstalk between the mass channels requires more care than for the high-resolution burst mode data. Some crosstalk between the water and solar wind mass groups can still occur, as further discussed in Sect. 3. The mass range of the instrument is divided into six different mass bins in normal mode. Occasionally, however, only data from three of them are transmitted to the ground, see Nilsson et al. (2007) for details.

\section{Observations}

\subsection{Data selection and treatment}

For this study we have separated the observed ions into solar wind ions and heavier ions of cometary origin.

Our high-resolution data indicate that these cometary ions are mostly water ions, therefore we refer to them as water ions throughout the text for convenience. We use both high-resolution and normal mode data, but we only use data with high postacceleration and only data where the complete mass channel range is transmitted to ground. In November and December 2014, an alternating post-acceleration mode was used, with every second complete angular scan having medium postacceleration. As we use only high post-acceleration data in this study, the number of data points per operated time is halved.

Crosstalk between mass channels can occur. This can significantly affect the results when the signal in one mass range is much stronger than in any other mass channel. Such crosstalk occurs independently for each energy and elevation angle interval, as these are obtained at different times. Most of the time, solar wind-originating ions and water ions are seen arriving from different directions. In the high mass resolution mode, any crosstalk between the mass ranges (broadening of the mass response due to a strong signal) can be identified. This is not fully possible in normal mode as the precise location of the signal on the detector plate is not known. For energies above $500 \mathrm{eV}$, the water ion signal is therefore set to zero if the signal in the solar wind mass channel range is greater than that in the water mass channel range. This is done individually for each energy level and elevation angle and is also applied to burst mode data to obtain a homogenous data set. Because some crosstalk occurs between neighboring sectors (see Fig. 1 for the definition of the sectors), we tested whether the solar wind signal is stronger than the water ion signal on the sum of all sectors. In this way, we avoided as much as possible falsely counting contamination from solar wind ions as real water ions. Similarly, there is some broadening of the mass response for strong water ion fluxes. We determined that so far, we have only seen a real signal in the solar wind mass range for energies below $100 \mathrm{eV}$ on a few occasions in February and March in the high-resolution data, therefore we set all these to zero.

From examining solar wind data far from the comet, it has been determined that sectors 0,2 , and 3 are prone to pick up crosstalk from an intense primary proton signal in any other sector. This can probably be compensated for, but we have chosen not to include these sectors in our analysis of the angular distributions of accelerated ions. These sectors cover a direction from 

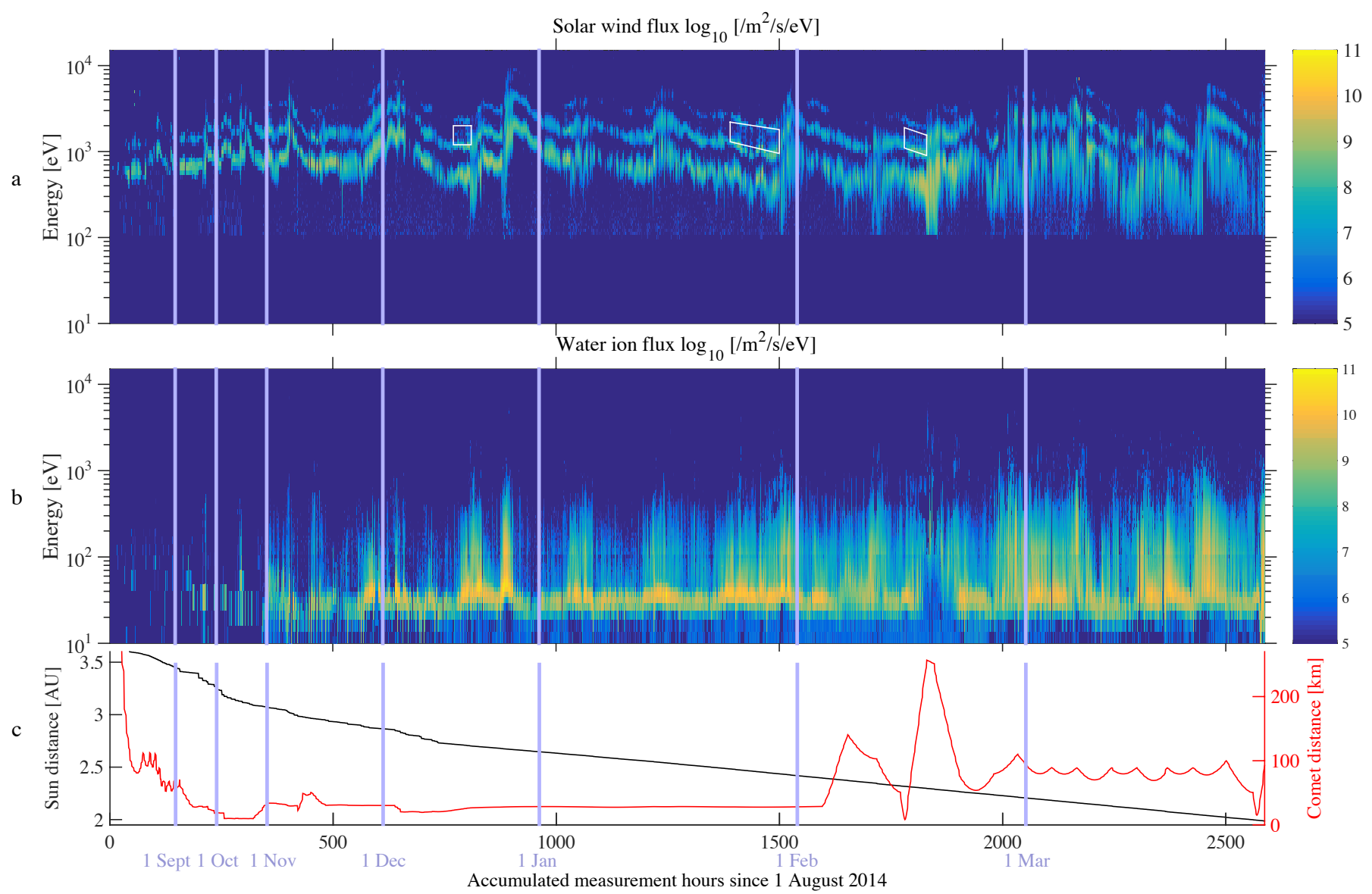

Fig. 2. Energy spectrograms summed over all viewing directions and integrated over one hour for a) solar wind ions and b) water group ions $\left[\mathrm{m}^{-2} \mathrm{~s}^{-1} \mathrm{eV}^{-1}\right]$. Panel c) shows the distance to the comet (red line, right $y$ axis, in km) and the heliocentric distance (black line, left $y$ axis, in AU). The blue vertical lines indicate the start of each month in the dataset. The $x$ axis shows accumulated hours of measurements since 1 August 2014. Note that the timescale is not linear since data gaps were removed. In panel a), the white transparent boxes indicate where $\mathrm{O}^{6+}(\mathrm{m} / q=2.7)$ ions were detected by RPC-ICA. They appear as a weak signal around $1.5 \mathrm{keV}$, between two stronger signals $\left(\mathrm{He}^{2+}\right.$ and $\left.\mathrm{He}^{+}\right)$.

which we usually do not see any signal except at the lowest energies (see Fig. 1 for illustration). Sector 1 suffers from another problem, it is very insensitive and thus not used. We have shaded these unused sectors in Fig. 1 to facilitate identifying them.

To save telemetry through more effective loss-less compression, a two-count background reduction is used. This was not always used for the early data. This is compensated for by instead subtracting background as part of the data analysis for the data without onboard subtraction.

\subsection{Overview of comet activity}

Figure 2 shows differential particle fluxes summed over all sectors and elevations in units of $\mathrm{m}^{-2} \mathrm{~s}^{-1} \mathrm{eV}^{-1}$ as a function of energy and time. This is the total flux in the angular range observed by RPC-ICA. The instrument was run sparingly before December 2014 and full time except during thruster firings from the beginning of 2015. Both for the efficient use of space and because the eye is easily fooled into interpreting data gaps as a signature of low activity, we show the data without data gaps. As a result, the timescale is not linear. Data have been integrated over $1 \mathrm{~h}$ intervals to obtain data points that are visible in the plot. The beginning of each month is indicated with a vertical blue line and the month name. Figure 2 a shows the energy distribution of the solar wind ions, while Fig. 2b shows the energy distribution of the water ions. Panel c shows the distance to the comet (black line) and the heliocentric distance of Rosetta (red line).

In panel a, a few features of interest can be seen. Typically, three rather continuous populations at different energies can be identified. The lowest energy population are the solar wind protons, with the solar wind alpha particles $\left(\mathrm{He}^{2+}\right)$ occurring at twice the energy per charge of the protons, as expected for the solar wind. At twice the energy per charge of $\mathrm{He}^{2+}$, a third population can be seen frequently. High-mass resolution data confirm that this is $\mathrm{He}^{+}$, created through charge exchange between solar wind alpha particles and the comet atmosphere. The ratio between the $\mathrm{He}^{+}$and $\mathrm{He}^{2+}$ fluxes is a direct function of the integrated comet atmosphere density through which the solar wind has passed. Occasionally, a fourth solar wind population can be seen even in this low-resolution plot, located in between the $\mathrm{He}^{2+}$ and $\mathrm{He}^{+}$populations. For example, around $800 \mathrm{~h}$, 1400-1500 h, and after $1800 \mathrm{~h}$ observation time, weak signals are indicated using white boxes. These ion signatures are consistent with high-charge state solar wind ions, in particular $\mathrm{O}^{6+}$; these high-charge state ions are known to play an important role in solar wind charge-exchange reactions, leading to UV and X-ray cometary emissions (Cravens 2002).

The solar wind energy is quite typical for the solar wind, ranging between $500 \mathrm{eV}$ and $2 \mathrm{keV}$, or 300 to $600 \mathrm{~km} \mathrm{~s}^{-1}$. The width of the proton energy distribution can be seen to increase with time in Fig. 2, although we note that temporal variations 


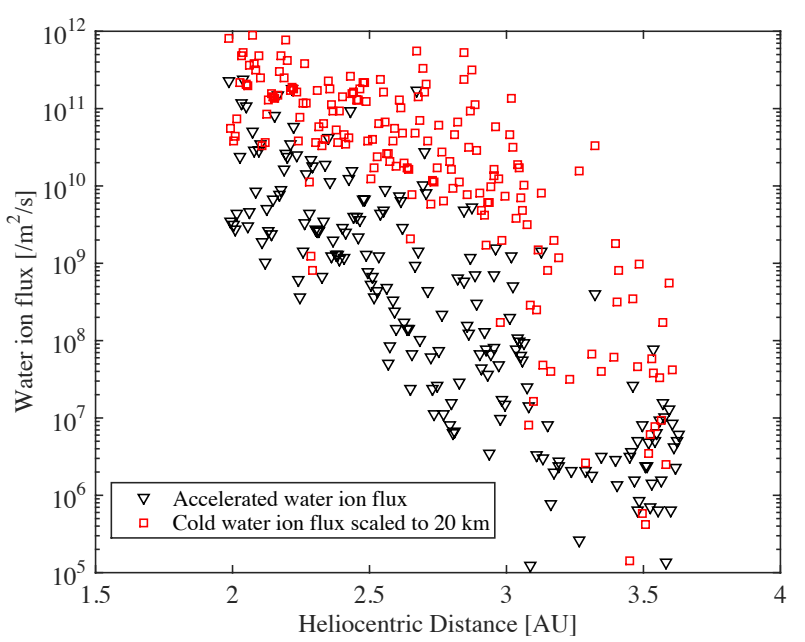

Fig. 3. Accelerated water ion flux integrated over the energy interval $120 \mathrm{eV}$ to $5 \mathrm{keV}$ and $24 \mathrm{~h}$ as a function of the heliocentric distance $[\mathrm{AU}]$ (black triangles). For reference, we also show the total integrated cold water ion flux, scaled to $20 \mathrm{~km}$ comet distance assuming an $r^{-1}$ fall-off with distance (red squares).

also widen the spectrogram in this 1-h-averaged plot. The flow direction of the solar wind ions deviates significantly from the anti-sunward direction, see Sect. 3.5. The deviation is an effect of the mass-loading caused by the addition of the comet ions to the solar wind flow (Nilsson et al. 2015). Toward the end of February, several cases of strong broadening of the proton energy spectra can be seen. This is further emphasized throughout March, when the solar wind energy spectra are generally broader. This could be due to the fact that the nature of the comet atmosphere-solar wind interaction is about to change and that the solar wind is significantly heated and slowed down.

In panel $b$, one can note that the water ions can be divided into a low-energy part with an energy of about 10 to $50 \mathrm{eV}$, which is more persistent, and they also have a higher flux density. We refer to this as the cold ion population. At higher energies, typically up to the proton energy, but sometimes higher, an accelerated water ion population can be seen frequently. The accelerated water ion flux is more sporadic, but from January onward, it is rather persistent. The low-energy population shows a higher variability from the beginning of February, when Rosetta left the bound orbits and the distance to the comet started to change considerably with time. The low-energy limit is expected to be a function of the spacecraft potential, which is typically negative close to the nucleus, see also Nilsson et al. (2015). The cold ion density frequently varies with the nucleus rotation, a case study of this is shown in Goldstein et al. (2015).

To even out any variability connected to the comet rotation period (12.40 h, see Mottola et al. 2014), it is suitable to examine data averaged over a time period of at least $12 \mathrm{~h}$. We have chosen to study a data set averaged over 24 h. In Fig. 3 we show the average flux $\left[\mathrm{m}^{-2} \mathrm{~s}^{-1}\right]$ of accelerated water ions (120 eV and above, black triangles) and of cold water ions (i.e., 10 to $50 \mathrm{eV}$, red squares) as measured during each $24 \mathrm{~h}$ period as a function of the heliocentric distance. The cold water ions were scaled to $20 \mathrm{~km}$ distance assuming an $r^{-1}$ dependence on the cometocentric distance. This distance dependence assumes that the neutral atmosphere is not significantly depleted due to loss of neutrals from ionization and is optically thin. If this is the case, the neutral density profile scales as $n \sim 1 / r^{2}$, and the total number of photo ions formed within a sphere of radius $r$ varies linearly with $r$. Since these photo ions move away from the nucleus (typically at the velocity of their parent neutrals) and if they are assumed not to be lost by any chemical processes, the cold water ion flux, expressed per unit area, will vary with $r \times 1 / r^{2} \sim 1 / r$.

As can be seen, the accelerated water ion flux increases by four orders of magnitude between 3.6 and 2.0 AU. The scaled cold water ion flux initially shows a similar tendency, until a heliocentric distance of about 3 AU. This agrees with the visual impression of the energy spectrogram shown in Fig. 2. Note that the cold water ion flux can be severely underestimated due to the limited field of view at low energy. If we disregard the data taken before 30 October 2014 as this was before the patch of the instrument, the cold water ion flux shows a weak trend of less than an order of magnitude, while the accelerated water ion flux increases by 2 orders of magnitude from 3 to $2.0 \mathrm{AU}$.

The evolution of the comet electron environment is shown in Clark et al. (2015), their Fig. 3. The evolution of the observed electron fluxes resembles the evolution of the cold ion flux seen with ICA, with low counts in August, followed by increasing flux in September and October. The electron flux appears to be higher still in November and December, but with less of a trend, consistent with the post-patch RPC-ICA data.

\subsection{Cold water ions}

We noted in Sect. 3.2 that the cometary ions can be divided into a cold and an accelerated (fast) population. The main distinguishing features are prevalence (the cold population is almost always present) and flow direction.

The velocity of the cold population is still significantly affected by the velocity of the parent neutral population. The outgassing velocity is in the range 400 to $800 \mathrm{~m} \mathrm{~s}^{-1}$, corresponding to an energy of $2-6 \mathrm{eV}$ for water ions. When the plasma is dense enough, the spacecraft potential is negative, approximately $-10 \mathrm{~V}$. This can be determined using the RPC-LAP instrument (Eriksson et al. 2007). The lowest energy seen by RPC-ICA is thus typically some $20 \mathrm{eV}$ or more. The angle of arrival for ions with an energy of a few $10 \mathrm{eV}$ can be expected to be strongly influenced by the neutral outgassing velocity and the spacecraft potential.

As discussed in Sect. 3.2, the ion flux can be expected to fall off linearly with cometocentric distance. Indeed, the scatter of the data points in Fig. 3 is smaller when a linear relation between flux and distance is assumed as compared to a $\sim 1 / r^{2}$ relation expected for the neutral density.

\subsection{Accelerated water ions}

The fast water ion population has been accelerated by the solar wind electric field and possibly other processes related to ion pick-up, such as charge separation of electric fields occurring due to the strong density gradients and different motions of ions and electrons. The ions do not come directly from the nucleus, and the higher the energy, the farther away one can expect the source region to be. There is thus no obvious way to scale the accelerated water ion population. Farther away we could be closer to the source of the more accelerated ions, but we would miss part of the ions produced closer to the comet. Figure 4 shows the accelerated water flux density $\left[\mathrm{m}^{-2} \mathrm{~s}^{-1}\right]$ as a function of linear time. We show an integration from $120 \mathrm{eV}$ to $5 \mathrm{keV}$. The upper limit was chosen to minimize noise by excluding mostly empty energy channels (see Fig. 2). There is significant variability, but a trend toward a higher minimum flux can be seen. The highest 


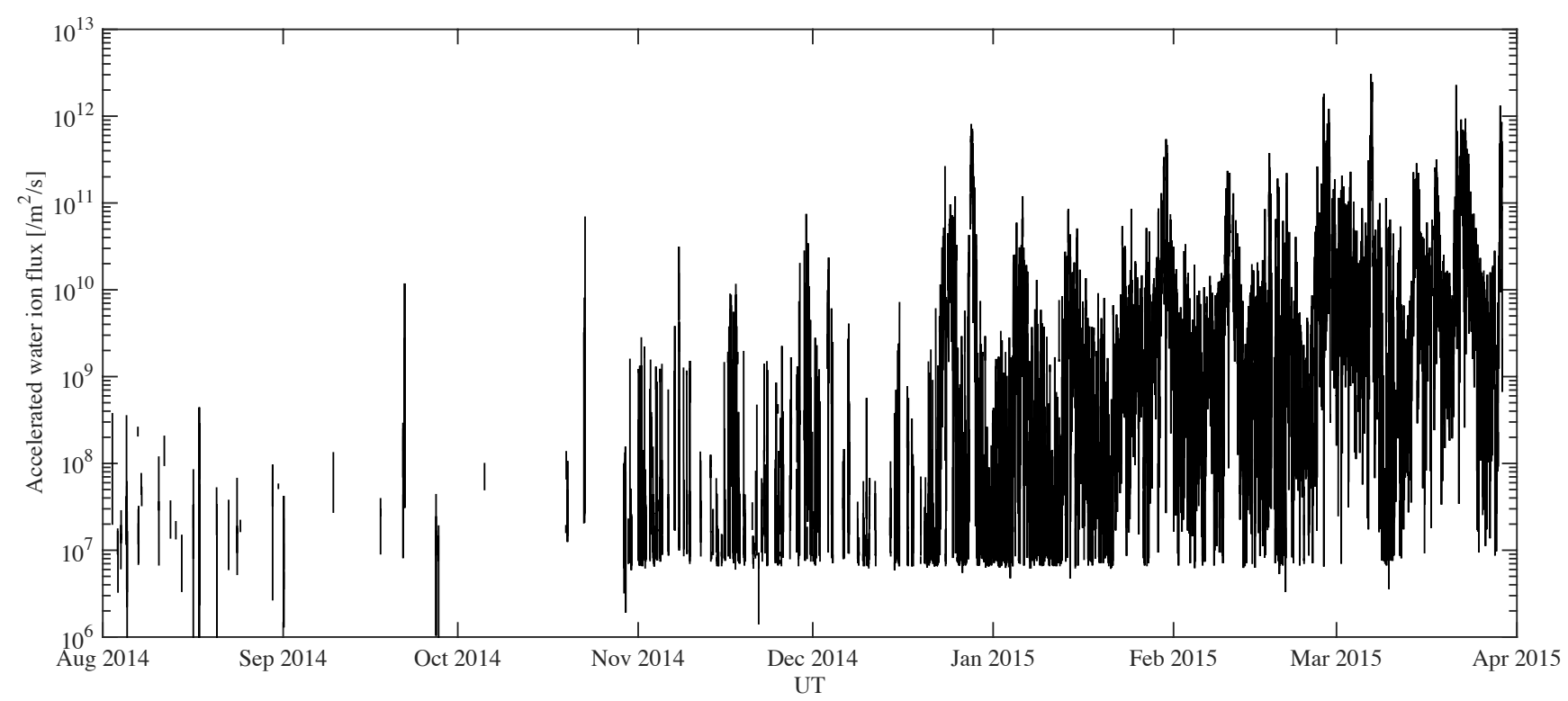

Fig. 4. Accelerated water ion flux integrated over the energy interval $120 \mathrm{eV}$ to $5 \mathrm{keV}$.
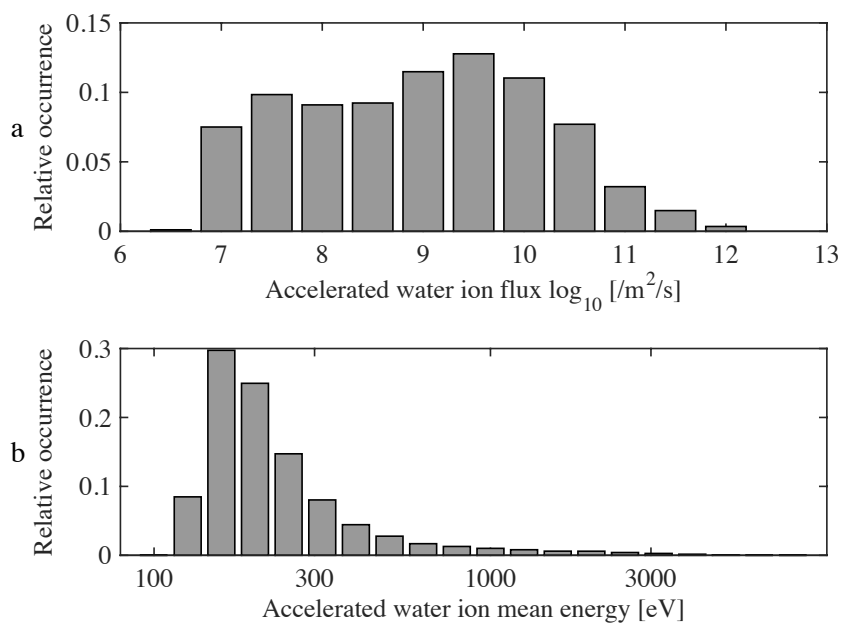

Fig. 5. a) Histogram of the relative occurrence of accelerated water ion flux integrated over the energy interval 120 to $5 \mathrm{keV}\left[\mathrm{m}^{-2} \mathrm{~s}^{-1}\right]$. b) Histogram over the relative occurrence for the mean accelerated water ion energy integrated over the entire instrument energy range $[\mathrm{eV}]$.

fluxes observed are also higher from November onward. This is most likely not a function of the upgraded energy table, which mainly affects the low-energy ions, but the much longer run time makes observations of high fluxes more likely as long as highflux events are sporadic. That the trend seen in Fig. 3 is stronger than that in Fig. 4 is explained by a higher occurrence of periods with high water ion flux. Figure 4 shows how the range of observed water ion flux values evolves with time.

The energetic ion environment is summarized in Fig. 5 with a histogram over the occurrence rate for observed accelerated water ion fluxes (panel a) and the average accelerated water ion energy (panel b). Regarding the temporal distribution, the histogram is statistically dominated by data from 2015, hence typical of a period with somewhat increased activity but before the formation of clear plasma boundaries. The flux of accelerated water ions is usually below a typical solar wind flux, which for these heliocentric distances is approximately $10^{11} \mathrm{~m}^{-2} \mathrm{~s}^{-1}$. Energies well above typical solar wind proton energies are sometimes seen (also seen in Fig. 2), although the mean energy typically is a few $100 \mathrm{eV}$.

\subsection{Flow directions}

The angular distribution of the ion flow may matter for determining whether the observed ions will also hit the surface of the nucleus. However, considering that the source region for fast ions detected by ICA probably is several $100 \mathrm{~km}$ distant, one may assume that, if ions reach Rosetta at typically $20-30 \mathrm{~km}$ from the nucleus center, they will also impact the nucleus. If the scale sizes involved are smaller than expected from simplest theory, then the flow direction could be more important for determining whether the observed fluxes will also hit the nucleus.

The ion flow direction is important for understanding how the cometary plasma tail forms and for elucidating the local photochemistry at play in the comet's environment. We therefore analyzed in which sectors the fluxes of the three main populations can be seen, that is, the solar wind, the accelerated water ions, and the cold water ions, and compared them with the sector pointing toward the Sun and the comet. To ensure the data quality, we only included solar wind data with a strong enough signal, at least 1000 counts integrated over all energies and elevation angles. For water ions, the criterion for the minimum number of counts was set to 100 . The use of a lower count threshold increases the general spread of the angular distribution. If water ion counts below ten counts are included, a peak occurs at $-90^{\circ}$ from the Sun direction for fast water ions. This is consistent with fast water ions moving along the solar wind convection electric field when there is no significant mass loading.

In Fig. 6 we show the angle of arrival away from the Sun with one sector $\left(22.5^{\circ}\right)$ resolution for solar wind ions (a), accelerated water ions (b), and cold water ions (c). Finally, we show in Fig. 6d the angle of arrival of cold water ions relative to the comet direction. We have taken the sector of the observed maximum minus the sector where the Sun/comet is located. Therefore a negative angle means that the ions were seen at the indicated angle clockwise from the Sun/comet direction in Fig. 1.

The solar wind ions are centered on the sector pointing toward the Sun, as expected. It is noteworthy that in more than $65 \%$ of the cases, the solar wind is deflected at least one sector, or $22.5^{\circ}$. The accelerated water ions show a similar distribution, but with a somewhat higher peak in the Sun direction. The cold water ions are seen approximately in between the Sun 

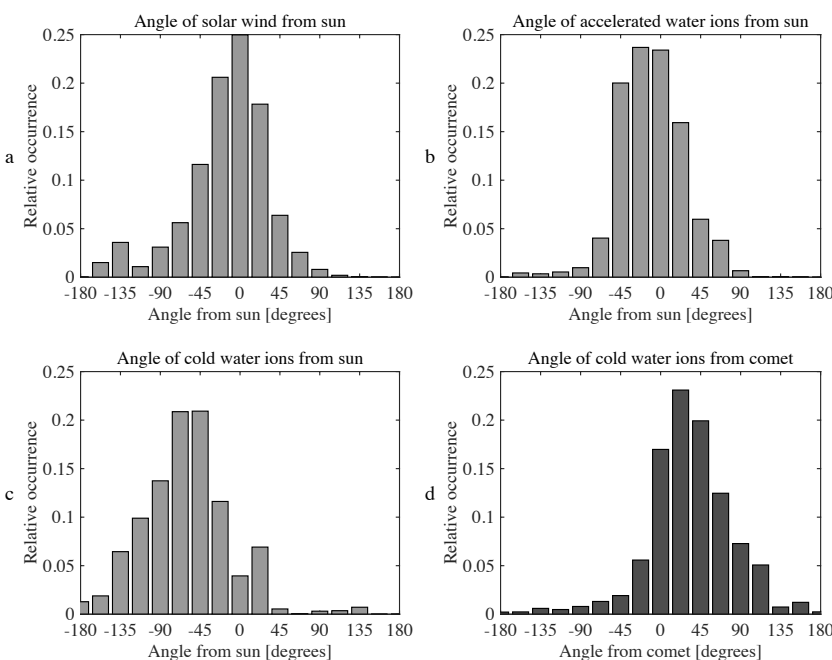

Fig. 6. Histograms of the relative occurrence of the three main populations seen by RPC-ICA with respect to the angle from the Sun direction. a) Solar wind ions. b) Accelerated water ions. c) Cold water ions. d) Cold water ions occurrences, shown here with respect to the angle from the comet. Each bin corresponds to one $22.5^{\circ}$ sector.

and the comet, similar to the case study shown in Nilsson et al. (2015). Note that we only studied the sectors here, there is typically some deflection beyond the sector plane as well. The elevations angles in an elevation sweep is different for different energies, however, and including this additional deflection is not trivial. We leave the full study of the deflection of the solar wind and the accelerated water ions as seen from RPC-ICA data to another study (Behar et al., in prep.). A detailed case study of solar wind deflection as seen using the RPC-IES instrument can be found in Broiles et al. (2015).

\section{Discussion}

The average accelerated water ion flux (120 eV to $5 \mathrm{keV})$ increased by four orders of magnitude when the heliocentric distance decreased from 3.6 to $2.0 \mathrm{AU}$, while the ionizing solar EUV flux and the solar wind flux increased by only a little more than a factor 2 . The change is most striking when comparing the excursions made in February 2015, out to $250 \mathrm{~km}$ distance from the comet, with the situation at a similar comet distance upon arrival in August 2014. In February strong fluxes of accelerated water ions are constantly present, in August they were rare sporadic events.

We can see in Fig. 2 that the water ion flux in each individual energy channel has not changed very much, but high fluxes are more common and seen over a wider energy range. In Fig. 4 the full resolution data show that the strongest change in the average flux arises because the high flux levels are more common with time/smaller heliocentric distance. To a first approximation, the energy range may correspond to the size of the source region, assuming a spatially extended acceleration. With this assumption, the larger the peak energy at the observation point, the longer the ions have traveled from the source. The strong increase in accelerated water ion fluxes thus shows that the source is spatially larger and more uniform with time. At the same time, data from the neutral gas spectrometer ROSINA (Balsiger et al. 2007) onboard Rosetta do not indicate a strong increase of the local neutral density. This is consistent with the relatively constant level of the low-energy flux seen by RPC-ICA for the time period after 30 October 2014, when the energy table was updated. There are some suggestions that comet dust particles can serve as a source of volatiles, an extended atmospheric source (Harris et al. 1997). This scenario might explain the increase of accelerated ions coming from the upstream direction while the local ion production does not show a similar increase.

The cold water ion population is distinct from the accelerated water ions in that the ions have a different angular distribution. If the ions were newly born and not affected by any electric fields, we would expect them to come from the direction of the nucleus. This is not the case: the typical angle of arrival of the cold water ions is in between the Sun and the comet nucleus, just as in the case study of Nilsson et al. (2015). An unperturbed solar wind electric field could not yield a flow in this direction because it would be perpendicular to the comet-Sun line. A possible explanation is that the actual electric field of the mass-loaded solar wind, which causes the deflection of the solar wind ions and the acceleration of the water ions, has a component in the anti-sunward direction. This would be consistent with the angular distribution of the accelerated water ions. A simple scenario would be that the pickup ions initially move along the undisturbed solar wind electric field, while the newly created electrons $E \times B$ drift, causing a charge separation. The electric field of that charge separation would initially have a component opposite to the solar wind electric field and a component in the anti-sunward direction. This is consistent with both the flow of the water ions and the observed deflection of the solar wind.

Another possibility that needs to be further investigated is whether a nonuniform spacecraft potential could yield a preferential angle of arrival of low-energy water ions. If this is the case, the angle of arrival should be centered around the same sector because this is the physical layout of the spacecraft, which is a prime factor in determining the angle of arrival. We note that the angular distribution relative to the comet shown in Fig. 6 is somewhat narrower than the range of physical sectors in which the cold water ions are typically seen (not shown), indicating that the spacecraft potential distribution is at least not the only factor determining the angle of arrival of the cold ions.

Sputtering of the comet nucleus by the solar wind may affect processes at the nucleus surface and affect the composition of the coma minor species (Wurz et al. 2015). Whereas the angle of the solar wind is different from that of an undisturbed solar wind, the flux remains similar. A large fraction of the solar wind will have charge-exchanged when passing through the comet atmosphere (Nilsson et al. 2015). It is noteworthy that the flux of water ions impinging on the nucleus is frequently of a similar order of magnitude as that of the solar wind. At times, it is even higher than a typical solar wind flux and reaches above $10^{11} \mathrm{~m}^{-2} \mathrm{~s}^{-1}$. The energy of the water ions is on average a few $100 \mathrm{eV}$, lower than that of the solar wind, while the momentum is expected to be similar, owing to the much higher mass of the water ions.

The electrodynamic environment of the comet will also affect small dust grains, nanograins. The RPC-IES instrument detects a signal that is very likely caused by nanograins (Gombosi et al. 2015). The signal is rather sporadic, which may be due to the solar wind electric field affecting the motion of the negatively charged dust grains. The electric field of the disturbed solar wind affecting the accelerated water ions will therefore also affect the trajectories of charged dust particles.

\section{Conclusions}

The flux of accelerated water ions moving from the upstream direction back toward the nucleus is a strongly nonlinear function 
of heliocentric distance. The accelerated water ion flux averaged over $24 \mathrm{~h}$ increases by possibly as much as four orders of magnitude between 3.6 and 2.0 AU. This is due to both a higher occurrence frequency of periods with high water ion flow and to a broadening in energy of the water ion flux. The latter indicates a larger spatial source region. The higher occurrence frequency indicates a more uniform source as a function of the comet rotation, latitude, and other factors that control the source density (neutral gas). It may also indicate a more uniform interaction of the solar wind and the atmosphere.

The accelerated water ions frequently reach a flux similar to that of the solar wind at a Sun distance of $2.0 \mathrm{AU}$, that is, about $10^{11} \mathrm{~m}^{-2} \mathrm{~s}^{-1}$, although a more typical value is lower than $10^{10} \mathrm{~m}^{-2} \mathrm{~s}^{-1}$.

The energetic water ions are typically accelerated to a few $100 \mathrm{eV}$, but sometimes to more than $1 \mathrm{keV}$. The momentum flux can typically be similar to that of the solar wind, as the kinetic energy and flux is lower but the mass much higher.

The solar wind is significantly deflected from the antisunward flow direction, typically by at least one sector $\left(22.5^{\circ}\right)$. The solar wind is not strongly decelerated throughout most of this period. Toward the end of February, at a Sun distance of about 2.3 AU, a strong broadening of the proton energy spectra started to occur sporadically and a more significant deceleration of the protons can be seen. RPC-ICA has so far detected the unambiguous signatures of energetic $\mathrm{H}^{+}$and $\mathrm{He}^{2+}$ ions and heavy ions such as $\mathrm{O}^{6+}$ of solar wind origin, as well as chargeexchanged $\mathrm{He}^{+}$ions.

Acknowledgements. Rosetta is a European Space Agency (ESA) mission with contributions from its member states and the National Aeronautics and Space Administration (NASA). Rosetta's Philae lander is provided by a consortium led by DLR, MPS, CNES and ASI. The work on RPC-ICA was funded by the Swedish National Space Board under contracts 108/12 and 112/13. Work at the Belgian Institute for Space Aeronomy was supported by the Belgian Science Policy Office through the Solar-Terrestrial Centre of Excellence and by
PRODEX/ROSETTA/ROSINA PEA 4000107705. CSW and EK are supported by the Academy of Finland. We acknowledge the staff of CDDP and IC for the use of AMDA and the RPC Quicklook database (provided by a collaboration between the Centre de Données de la Physique des Plasmas (CDPP) supported by CNRS, CNES, Observatoire de Paris and Université Paul Sabatier, Toulouse and Imperial College London, supported by the UK Science and Technology Facilities Council). We are indebted to the whole Rosetta mission team, Science Ground Segment and Rosetta Mission Operation Control for their hard work that makes this mission possible.

\section{References}

Alfvén, H. 1957, Tellus, IX, 92

Balsiger, H., Altwegg, K., Bochsler, P., et al. 2007, Space Sci. Rev., 128, 745

Biermann, L. 1951, Z. Astrophys., 29, 274

Broiles, T. W., Burch, J. L., Clark, G., et al. 2015, A\&A, 583, A21

Burch, J., Goldstein, R., Cravens, T., et al. 2007, Space Sci. Rev., 128, 697

Clark, G., Broiles, T. W., Burch, J. L., et al. 2015, A\&A, 583, A24

Coates, A. 1997, Adv. Space Res., 20, 255

Coates, A. J., Johnstone, A. D., Wilken, B., Jockers, K., \& Glassmeier, K.-H. 1989, J. Geophys. Res., 94, 9983

Cravens, T. E. 2002, Science, 296, 1042

Cravens, T., \& Gombosi, T. 2004, Adv. Space Res., 33, 1968

Eriksson, A. I., Boström, R., Gill, R., et al. 2007, Space Sci. Rev., 128, 729

Glassmeier, K.-H., Boehnhardt, H., Koschny, D., Kührt, E., \& Richter, I. 2007, Space Sci. Rev., 128, 1

Goldstein, R., Burch, J. L., Mokashi, P., et al. 2015, Geophys. Res. Lett., 42, 3093

Gombosi, T. I., Burch, J. L., \& Horányi, M. 2015, A\&A, 583, A23

Harris, W. M., Combi, M. R., Honeycutt, R. K., Mueller, B. E. A., \& Scherb, F. 1997, Science, 277, 676

Mottola, S., Lowry, S., Snodgrass, C., et al. 2014, A\&A, 569, L2

Neugebauer, M. 1990, Rev. Geophys., 28, 231

Nilsson, H., Lundin, R., Lundin, K., et al. 2007, Space Sci. Rev., 128, 671

Nilsson, H., Stenberg Wieser, G., Behar, E., et al. 2015, Science, 347, 0571

Richardson, I. G., Cowley, S. W. H., Hynds, R. J., et al. 1987, Planet. Space Sci., 35,1323

Richter, I., Koenders, C., Auster, H.-U., et al. 2015, Geophys. Res. Lett., submitted [arXiv: 1505.06068]

Rubin, M., Koenders, C., Altwegg, K., et al. 2014, Icarus, 242, 38

Szegö, K., Glassmeier, K.-H., Bingham, R., et al. 2000, Space Sci. Rev., 94, 429

Wurz, P., Rubin, M., Altwegg, K., et al. 2015, A\&A, 583, A22 